\title{
Efeito da Extrusão nas Propriedades Bioquímicas e Tecnológicas da Farinha Bandinha de Feijão
}

\author{
Christiane A. Starling, Fernanda G. de Souza \& \\ Adriana A. de Carvalho
}

A bandinha de feijão é um subproduto da indústria de processamento de feijão. $\mathrm{O}$ objetivo deste trabalho foi avaliar os efeitos das condições de extrusão no que se refere a fatores bioquímicos e propriedades tecnológicas de bandinha de feijão. As bandinhas foram trituradas e a farinha obtida foi extrusada. Tanto a farinha crua e extrusada foram testadas quanto: ao conteúdo de inibidores de tripsina, inibidores de $\alpha$-amilase, atividade hemaglutinante, às propriedades de solubilidade e absorção de água, à atividade emulsificante e estabilidade da emulsão. Os resultados evidenciaram que a extrusão eliminou os fatores antinutricioais e melhorou as propriedades tecnológicas.

Palavras-chave: bandinha de feijão; fatores antinutricionais; propriedades tecnológicas.

The little band bean is a byproduct of industrial processing beans. The objective of this study was to evaluate the effect of extrusion conditions with regard to biochemical factors and technological properties of bean little band. The little band was crushed and the flour obtained was extruded. Both raw and extruded flour were tested: the content of trypsin inhibitors , $\alpha$ - amylase inhibitors, hemagglutinating activity, the solubility properties and water absorption, the emulsifying activity and emulsion stability. The results showed that the extrusion eliminated antinutritional factors and improved technological properties.

Keywords: bean little band; antinutritional factors; technological properties. 


\section{Introdução}

$\mathrm{Na}$ sociedade atual, os benefícios nutricionais dos alimentos estão em alta. Os consumidores exigem, além de sabor agradável e praticidade, alto valor nutritivo, baixos teores calóricos, fibras e outros benefícios à saúde em alimentos industrializados. Estes fatores fazem com que as indústrias ofereçam produtos mais nutritivos. Atentos a essa realidade, a empresa IBIÁ, por meio do Edital SENAI SESI de Inovação, desenvolveu, com o apoio técnico científico do SENAI (Serviço Nacional de Aprendizagem Industrial) e SESI (Serviço Social da Indústria) ambas instituições do Sistema FIEG (Federação das Indústrias do Estado de Goiás), um projeto de inovação tecnológica e social para lançar no mercado um produto inovador: a farinha extrusada de bandinha de feijão.

A bandinha de feijão (grão de partido ao meio) é um subproduto da indústria de beneficiamento do feijão. Apesar de ser um grão fracionado seus aspectos nutricionais são mantidos. Entretanto, o seu baixo valor comercial inviabiliza a sua comercialização direta. Dessa forma, o aproveitamento industrial desses subprodutos como matéria-prima na obtenção de produtos alimentícios torna-se uma alternativa viável, uma vez que apresentam qualidade nutricional semelhante à dos grãos inteiros.

O feijão é uma leguminosa amplamente cultivada no Brasil e consequentemente um dos maiores consumidores $^{1}$. Por ser um alimento de significante valor nutricional ${ }^{2}$ assume enorme importância na alimentação humana e, além disso, tem aceitação nos mais diferentes hábitos alimentares ${ }^{3}$. Entretanto ele apresenta fatores antinutricionais que, para eliminá-los torna-se necessário o emprego de tratamento térmico antes de seu consumo ${ }^{4}$.

Um dos tratamentos que tem sido empregado para o processamento do feijão é a extrusão termoplástica ${ }^{5}$.

Este tratamento pode ser definido como o processo contínuo no qual o cisalhamento mecânico é combinado com calor para promover a gelatinização do amido e desnaturação das proteinas, resultando em sua plasticidade e reestruturação para obtenção de produtos com novas características tecnológicas e novos formatos ${ }^{6}$. A gelatinização do amido resulta do aumento da absorção de água, permitindo também a proteólise mais extensiva e favorecendo a digestibilidade das proteínas ${ }^{4}$. Isso porque durante o processo de extrusão ocorrem muitas reações como destruição e modificação dos aminoácidos por oxidação o que retardar sua liberação durante o processo digestivo. A extrusão também pode inativar enzimas e eliminar fatores antinutricionais do feijão ${ }^{7}$.

Diante deste contexto, este projeto teve como objetivo avaliar os efeitos das condições de extrusão no que se refere a fatores antinutricionais e propriedades tecnológicas da farinha da bandinha de feijão e partir daí industrializá-la. comum da variedade carioca. Além disso, o projeto prevê a inclusão desta farinha extrusada como ingrediente principal para formulações alimentícias de preparo instantâneo e de cozimento rápido, a serem incorporadas ao cardápio da população, como alternativa de aumento no aporte proteico da dieta.

\section{Materiais e Métodos}

\section{PROCESSAMENTO DA BANDINHA DE FEIJÃO}

As bandinhas de feijão (Phaseolus vulgaris) foram triturados em moinho de facas tipo Willey modelo EDB-5, peneirados em tamis de 35 mesh $(0,425 \mathrm{~mm})$, e sua umidade foi ajustada conforme planejamento descrito nas Tabelas 1, 2 e 3 e armazenadas por 24 horas em refrigerador para estabilização dos valores de umidade. Depois as farinhas foram submetidas ao processo de extrusão em uma extrusora de parafuso simples (Inbramaq, PQ-30, Ribeirão Preto-SP, Brasil) com taxa de compressão 3:1, matriz com duas entradas, camisa helicoidal, subtrafilas de $4 \mathrm{~mm}$ e trafilas de $4 \mathrm{~mm}$. A rotação utilizada no equipamento foi de 150 rpm.

A extrusão foi realizada utilizando temperatura central de extrusão de $150{ }^{\circ} \mathrm{C}^{8}$. Os extrusados foram secos à temperatura ambiente, moídos em moinho de facas tipo Willey modelo EDB-5 peneirados em tamis de $0,425 \mathrm{~mm}$, acondicionados em sacos de polietileno e refrigerados $\left(4^{\circ} \mathrm{C}\right)$. 
Tabela 1. Conteúdo de inibidores enzimáticos encontrados na farinha de bandinha de feijão carioca crua e extrusada (Planejamento 1).

\begin{tabular}{|c|c|c|c|c|c|}
\hline & Temperatura ${ }^{\circ} \mathrm{C}$ & Umidade $(\% *)$ & Inibidor de Tripsina & $\begin{array}{c}\text { Inibidor de } \\
\alpha \text {-amilase }\end{array}$ & $\begin{array}{c}\text { Atividade } \\
\text { hemaglutinante }\end{array}$ \\
\hline 1 & 90 & 10 & $1,47(63.88 \%)$ & $0,23(57,33 \%)$ & \\
\hline 2 & 90 & 16 & $2,29(43,73 \%)$ & $0,04(92,32 \%)$ & \\
\hline 3 & 110 & 10 & $1,24(69,53 \%)$ & $0,20(63,86 \%)$ & \\
\hline 4 & 110 & 16 & $2,14(47,42 \%)$ & $0,0(100 \%)$ & \\
\hline 5 & 85,86 & 13 & $1,74(57,25 \%)$ & $0,10(82,31 \%)$ & \\
\hline 6 & 114,14 & 13 & $1,23(69,78 \%)$ & $0,12(78,85 \%)$ & \\
\hline 7 & 100 & 8,76 & $0,09(97,79 \%)$ & $0,0(100 \%)$ & \\
\hline 8 & 100 & 17,24 & $1,18(71,01 \%)$ & $0,07(86,89 \%)$ & \\
\hline 9 & 100 & 13 & $1,28(68,55 \%)$ & $0,13(75,65 \%)$ & \\
\hline 10 & 100 & 13 & $1,1(72,97 \%)$ & $0,15(72,04 \%)$ & \\
\hline \multicolumn{3}{|c|}{ Farinha crua } & 4,07 & 0,55 & \\
\hline
\end{tabular}

Os dados apresentados correspondem a media de três determinações. Os valores entre parênteses correspondem à redução ocasionada no conteúdo de inibidores em cada um dos tratamentos. (*) Dada em base seca.

Tabela 2. Conteúdo de inibidores enzimáticos encontrados na farinha extrusada após fervura por 5 minutos (Planejamento 1).

\begin{tabular}{|c|c|c|c|c|}
\hline & Temperatura ${ }^{\circ} \mathbf{C}$ & Umidade $\left(\mathbf{\%}^{*}\right)$ & Inibidor de Tripsina & Inibidor de $\boldsymbol{\alpha}$-amilase \\
\hline 1 & 90 & 10 & $1,41(65,09 \%)$ & $0,20(61,54 \%)$ \\
\hline 2 & 90 & 16 & $2,21(45,29 \%)$ & $0,0(100 \%)$ \\
\hline 3 & 110 & 10 & $1,22(69,80 \%)$ & $0,12(76,92 \%)$ \\
\hline 4 & 110 & 16 & $2,11(47,77 \%)$ & $0,0(100 \%)$ \\
\hline 5 & 85,86 & 13 & $1,72(57,43 \%)$ & $0,0(100 \%)$ \\
\hline 6 & 114,14 & 13 & $1,21(70,04 \%)$ & $0,03(94,23 \%)$ \\
\hline 7 & 100 & 8,76 & $0,05(98,76 \%)$ & $0,0(100 \%)$ \\
\hline 8 & 100 & 17,24 & $1,12(72,28 \%)$ & $0,25(51,92 \%)$ \\
\hline 9 & 100 & 13 & $1,20(70,29 \%)$ & $0,08(84,61 \%)$ \\
\hline 10 & 100 & 13 & $1,11(72,52 \%)$ & $0,21(59,61 \%)$ \\
\hline
\end{tabular}

Os dados apresentados correspondem a media de três determinações. Os valores entre parênteses correspondem à redução ocasionada no conteúdo de inibidores em cada um dos tratamentos.

Tabela 3. Conteúdo de inibidores enzimáticos encontrados na farinha de bandinha de feijão carioca crua e extrusada (Planejamento 2).

\begin{tabular}{|c|c|c|c|c|}
\hline & Temperatura $^{\circ} \mathbf{C}$ & Umidade (\%*) & Inibidor de Tripsina & Inibidor de $\boldsymbol{\alpha}$-amilase \\
\hline 1 & 110 & 19 & $0,0(100 \%)$ & $0,0(100 \%)$ \\
\hline 2 & 120 & 19 & $0,0(100 \%)$ & $0,0(100 \%)$ \\
\hline 3 & 120 & 16 & $0,0(100 \%)$ & $0,0(100 \%)$ \\
\hline 4 & 110 & 13 & $0,15(94,79 \%)$ & $0,0(100 \%)$ \\
\hline \multicolumn{2}{|c|}{ Farinha crua } & 2,88 & 3,43 & \\
\hline
\end{tabular}

Os dados apresentados correspondem a media de três determinações. Os valores entre parênteses correspondem à redução ocasionada no conteúdo de inibidores em cada um dos tratamentos 


\section{FATORES ANTINUTRICIONAIS}

As farinhas foram utilizadas para a avaliação da presença de inibidores enzimáticos: tripsina e $\alpha$-amilase.

\section{Inibidores de tripsina}

$\mathrm{O}$ ensaio de inibição foi realizado de acordo com metodologia descrita por Kunitz e modificada por Arnon ${ }^{10}$. A leitura da absorbância do sobrenadante foi realizada em comprimento de onda de $280 \mathrm{~nm}$ utilizando cubeta de quartzo. Para isso, extratos a $1 \%(\mathrm{p} / \mathrm{v})$ das farinhas foram preparados em tampão fosfato de sódio $0,1 \mathrm{~mol} \mathrm{~L}^{-1} \mathrm{pH} 7,6$, sob agitação a $4^{\circ} \mathrm{C}$ por $30 \mathrm{~min}$, seguido de centrifugação por 10 min a 4000 g. Para expressão dos resultados foi definido que uma unidade de enzima (UE) é a quantidade suficiente para provocar alteração de 0,1 na absorbância a $280 \mathrm{~nm}$. A unidade de inibição foi determinada pela diferença entre a atividade observada sem a presença de inibidores e aquela observada após a adição de extrato contendo inibidores.

\section{Inibidores de a-amilase}

$\mathrm{O}$ ensaio de inibição foi realizado de acordo com metodologia descrita por Deshpande et $\mathrm{al}^{11}$. Para isso, extratos a $1 \%(\mathrm{p} / \mathrm{v})$ das farinhas foram preparados em tampão acetato de sódio $0,1 \mathrm{~mol} \mathrm{L-1} \mathrm{pH} 5,0$, sob agitação a $4{ }^{\circ} \mathrm{C}$ por $30 \mathrm{~min}$, centrifugados a $4000 \mathrm{~g}$ por 10 min e o sobrenadante utilizado nos experimentos. Após as reação do extrato com $\alpha$-amilase (Novozymes), foram adicionados $100 \mu \mathrm{L}$ de solução de amido (Sigma Aldrich) 0,5\% (p/v) em tampão acetato de sódio 0,1 mol L-1 pH 5,0 e a solução foi incubada por 15 min a 40 ${ }^{\circ} \mathrm{C}$. Após a reação, o teor de açúcar redutor foi determinado segundo metodologia de Miller ${ }^{12}$. O teor de açúcar redutor foi determinado a partir de uma curva padrão de glicose. Determinou-se a atividade inibitória pela diferença entre a atividade da enzima sem a presença de inibidores em que uma unidade de enzima foi determinada como a quantidade (em micromols) de açúcar redutor produzido por mililitro de amostra em um minuto de reação.

\section{Atividade hemaglutinante}

A atividade hemaglutinante foi determinada de acordo com o método descrito por Moreira e Perrone ${ }^{13}$, usando suspensão de hemácias de coelho a $2 \%$ (v/v) em solução salina 0,15 mol L hemaglutinação foi definida como sendo o inverso da maior diluição de uma dada solução que ainda é capaz de aglutinar uma suspensão de hemácias a $2 \%$ (v/v).

\section{Determinação da digestibilidade in vitro de amigo proteína.}

A digestibilidade in vitro do amido foi determinada utilizando metodologia modificada de Batista et al.8. Para isso $200 \mathrm{mg}$ de farinha foram suspensos em $5 \mathrm{~mL}$ de solução de $\alpha$-amilase (Novozymes) $1 \%$ em tampão acetato $0,1 \mathrm{~mol} \mathrm{L-1} \mathrm{pH} 5,5$. O sistema foi incubado a $55{ }^{\circ} \mathrm{C}$ por $15 \mathrm{~min}$. Os tubos foram, então, resfriados à temperatura ambiente e o $\mathrm{pH}$ ajustado para 5,0, utilizandose solução de $\mathrm{HCl} 1$ mol L-1. Em seguida, foram adicionados $50 \mu \mathrm{L}$ de amiloglicosidade (Novozymes) e o sistema foi incubado a $50{ }^{\circ} \mathrm{C}$ por $2 \mathrm{~h}$. A quantidade de glicose liberada foi determinada utilizando kit comercial de determinação de glicose por glucose-oxidase (Gold Analisa Diagnóstica) e a porcentagem de amido digerível foi calculada utilizando-se a Equação (1):

Digestibilidade de amido $(\%)=\frac{\text { (Conteúdo de glicose liberada) } \times 0,9}{\text { Conteúdo de amido total }} \times 100$

Equação (1)

Por fim, o conteúdo de amido resistente foi calculado pela diferença entre o amido total e o amido digerível.

A digestibilidade proteica in vitro foi determinada usando a técnica multienzimática descrita por Hsu et al. ${ }^{14}$. Um grama de amostra foi disperso em $20 \mathrm{~mL}$ de tampão fosfato de sódio 0,1 mol L-1 pH 8,0 e incubado a $37^{\circ} \mathrm{C}$ por $10 \mathrm{~min}$. Em seguida, adicionou-se $0,5 \mathrm{~mL}$ de solução de tripsina ( $8 \mathrm{mg} \mathrm{mL}-1)$ e $0,5 \mathrm{~mL}$ de solução de pancreatina (20 mg mL-1), ambas preparadas em tampão fosfato $0,1 \mathrm{~mol} \mathrm{~L}-1 \mathrm{pH} 8,0$. O pH do sistema foi ajustado para 8,0 e volume completado para $25 \mathrm{~mL}$. A mistura foi então incubada a $37{ }^{\circ} \mathrm{C}$ por $10 \mathrm{~min}$, sob agitação. Após a hidrólise, as amostras foram resfriadas à temperatura ambiente e o pH foi medido com auxílio de um pHmetro. A redução observada no $\mathrm{pH}$ corresponde à presença de grupos carboxílicos de aminoácidos liberados pela hidrólise enzimática e a digestibilidade foi determinada utilizando-se a Equação (2):

Digestibilidade protéica $(\%)=425,68-47,64(\mathrm{pH})$

$$
\text { Equação (2) }
$$




\section{PROPRIEDADES TECNOLÓGICAS}

\section{Solubilidade e absorção de água}

O teste de absorção de água foi realizado utilizando 100 mg de farinha, pesados em tubos de plástico com tampa com a adição de $5 \mathrm{~mL}$ de água destilada. Na sequência, os tubos foram agitados por 1 minuto e centrifugados a $4000 \mathrm{~g}$ por 20 minutos. Em seguida, o sobrenadante foi retirado para determinação da solubilidade em água e o tubo contendo o precipitado foi novamente pesado ${ }^{15}$. A absorção de água foi determinada pela diferença de peso da amostra antes e após a absorção de água e o índice de absorção de água (IAA) foi calculado de acordo com a Equação (3):

Absorção de água $(\mathrm{g} / \mathrm{g})=\frac{\text { Peso do tubo após centrifugação }(\mathrm{g})-\text { Peso do tubo vazio }(\mathrm{g})}{\text { Peso da amostra }(\mathrm{g})}$

\section{Equação (3)}

O sobrenadante das amostras foi transferido para placas de Petri e colocadas em estufa a $105^{\circ} \mathrm{C}$ para secagem até peso constante. Em seguida, estas foram transferidas para dessecadores para atingirem temperatura ambiente e por fim foram pesadas em balança analítica. A solubilidade em água (SA) foi determinada pela diferença de peso do resíduo seco do sobrenadante (resíduo de evaporação) e o peso inicial da amostra, de acordo com a fórmula:

\section{Atividade emulsificante e estabilidade da emulsão}

Em tubos de plástico com tampa, foram adicionados $500 \mathrm{mg}$ de amostra, $5 \mathrm{~mL}$ de óleo de soja e $5 \mathrm{~mL}$ de água destilada. Depois foram agitados por $2 \mathrm{~min}$. Após a emulsificação, os tubos foram centrifugados a 3000 $\mathrm{g}$ por $5 \mathrm{~min}^{16}$. O cálculo da atividade emulsificante foi realizado de acordo com a Equação (4):

Solubilidade em água $(\%)=\frac{\text { Resíduo de evaporação }(\mathrm{g})}{\text { Peso da } \operatorname{amostra~}(\mathrm{g})} \times 100$

\section{Equação (4)}

\section{ANÁLISES FÍSICO-QUÍMICAS}

\section{Composição centesimal e valor calórico da farinha extrusada da bandinha de feijão}

A umidade foi determinada por aquecimento direto em estufa a $105^{\circ} \mathrm{C}$ por 24 horas. As cinzas foram determinadas por meio da calcinação das amostras em mufla a temperatura de $550^{\circ} \mathrm{C}$ até o peso constante (aproximadamente 5 horas). $\mathrm{O}$ teor de nitrogênio total foi quantificado e o teor de proteína bruta foi obtido pelo uso do fator 5,75 para conversão de nitrogênio em proteína ${ }^{17}$. A determinação de lipídios foi realizada de acordo com a $\mathrm{IAL}^{18}$. A determinação dos teores de fibra alimentar insolúvel, solúvel e a total foi realizada pelo método conforme descrito pela AOAC17 (2005) e por Prosky et al. ${ }^{19}$. A determinação de carboidratos foi realizada por diferença, por meio da seguinte fórmula: 100 - $(\mathrm{g} / 100 \mathrm{~g}$ umidade $+\mathrm{g} / 100 \mathrm{~g}$ cinzas $+\mathrm{g} / 100 \mathrm{~g}$ lipídios totais + $\mathrm{g} / 100 \mathrm{~g}$ proteína $+\mathrm{g} / 100 \mathrm{~g}$ de fibra alimentar total).

O valor calórico da amostra foi calculado pela soma das porcentagens de proteína e carboidratos multiplicados pelo fator $4(\mathrm{kcal} / \mathrm{g})$ somado ao teor de lipídios totais multiplicado pelo fator $9(\mathrm{kcal} / \mathrm{g})^{20,21,22}$.

\section{Composição de ácidos graxos, vitaminas e minerais da} farinha extrusada da bandinha de feijão.

Uma amostra da farinha extrusada da bandinha de feijão foi encaminhada ao Instituto de Tecnologia de Alimentos (ITAL) em Campinas-SP para determinação de ácidos $\operatorname{graxos}^{23,24,25,26,27}$, vitaminas ${ }^{28,29,30,31,32,33,34} \mathrm{e}$ minerais $^{35,36}$.

\section{Atividade de água da farinha extrusada da bandinha de feijão}

Uma amostra de farinha extrusada foi inserida no compartimento do analisador de atividade de água AquaLab modelo 4TE . O resultado foi obtido por leitura direta.

\section{Viscosidade da farinha extrusada da bandinha de feijão}

A análise de viscosidade foi conduzida em viscosímetro Brookfield modelo LVDV-II. Uma solução $10 \%(\mathrm{p} / \mathrm{v})$ de farinha de bandinha de feijão extrusada foi aquecida por 15 minutos a $90^{\circ} \mathrm{C}$. Após o aquecimento, a solução foi resfriada à temperatura ambiente e a viscosidade determinada. 


\section{ANÁLISE MICROBIOLÓGICA}

Para avaliação da qualidade microbiológica, a farinha extrusada da bandinha de feijão foi analisada segundo a RDC 12/200137, 38, 39, 40, 41,42.

\section{Resultados e Discussão}

\section{FATORES ANTINUTRICIONAIS}

Como pode ser observado na Tabela 1, o delineamento utilizado para a extrusão das farinhas não foi eficaz em eliminar os inibidores de tripsina. As condições de extrusão empregadas não foram suficientemente drásticas para desnaturar os inibidores e eliminar sua atividade antinutricional. Tais resultados podem ser explicados em função da alta estabilidade térmica dos inibidores de tripsina. Neste caso, a combinação de calor, pressão e umidade no processo de extrusão reduziu significativamente o conteúdo de inibidores, mas, não foi capaz de zerar sua atividade. Com relação aos inibidores de $\alpha$-amilase, a eliminação completa da atividade foi observada nos tratamentos 4 e 7 (Tabela 1). Nestes casos, a umidade foi o fator que mais interferiu na redução dos inibidores. Os testes de lectina na farinha de bandinha de feijão foram positivos, enquanto que na farinha extrusada, a atividade hemaglutinante foi completamente eliminada (Tabela 1). O binômio temperatura/umidade utilizado no processo de extrusão levou à eliminação da atividade hemaglutinante por desnaturação.

Sabendo-se que a qualidade dos feijões é limitada pela presença de fatores antinutricionais, a completa inativação e/ou remoção destes compostos através do processo de extrusão é uma alternativa para melhorar a qualidade nutricional deste grão, principalmente, no que se refere à biodisponibilidade de proteínas e carboidratos.
Como os resultados obtidos no planejamento não foram satisfatórios, quanto à eliminação dos inibidores enzimáticos, as farinhas foram submetidas a testes de maceração em água fervente por 5 minutos, uma vez que sua possível aplicação está relacionada com a introdução destas farinhas em preparações quentes. Desse modo, caso os inibidores sejam eliminados após a fervura, o residual de inibidores presente nas farinhas extrusadas não afetaria a qualidade nutricional do produto final. Os resultados do conteúdo de antinutricionais remanescentes nas farinhas extrusadas, após fervura por 5 minutos, estão apresentados na Tabela 2. A fervura reduziu consideravelmente o conteúdo de inibidores de $\alpha$-amilase, entretanto, teve pouca interferência no conteúdo de inibidores de tripsina.

Com base nestes resultados, foram propostas novas condições de extrusão (Tabela 3) no intuito de promover completa eliminação dos inibidores de tripsina e $\alpha$-amilase das amostras. Os resultados demonstraram que a temperatura e umidade utilizadas no processo de extrusão foram eficientes em eliminar a atividade inibitória presente na farinha extrusada em praticamente todos os tratamentos. Tal efeito pode ser explicado em função da desnaturação das moléculas de inibidores que, perdem sua estrutura tridimensional e, consequentemente, sua atividade antinutricional. $\mathrm{O}$ tratamento 4, por apresentar inibidores de tripsina remanescentes, após tratamento de extrusão, não foi utilizado para a realização dos testes de caracterização tecnológica e funcional.

A eliminação dos inibidores enzimáticos aumenta a qualidade nutricional das farinhas produzidas, aumentando a digestibilidade de proteínas e amido (Tabela 4). Além da eliminação dos inibidores, o efeito do calor e umidade ocasionou a abertura do grânulo de amido e a desnaturação proteica, o que contribui para o aumento da disponibilidade de sítios para o ataque enzimático, contribuindo para uma maior digestibilidade.

Tabela 4. Digestibilidade in vitro de amido e proteínas na farinha de bandinha de feijão carioca crua e extrusada.

\begin{tabular}{|c|c|c|c|c|}
\hline & Temperatura ${ }^{\circ} \mathbf{C}$ & Umidade (\%*) & Digestibilidade de amido (\%) & Digestibilidade de proteinas (\%) \\
\hline 1 & 110 & 19 & $88,28(54,09 \%)$ & $91,93(75,14 \%)$ \\
\hline 2 & 120 & 19 & $76,87(62,11 \%)$ & $94,28(73,27 \%)$ \\
\hline 3 & 120 & 16 & $78,61(60,74 \%)$ & $91,11(75,82 \%)$ \\
\hline \multicolumn{2}{|c|}{ Farinha crua } & 47,75 & 69,08 & \\
\hline
\end{tabular}

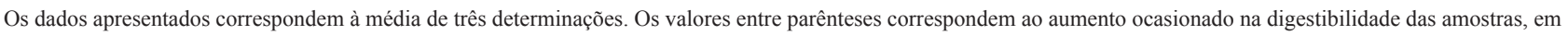
relação à farinha crua. 


\subsection{PROPRIEDADES TECNOLÓGICAS}

Os valores de absorção e solubilidade em água das farinhas extrusadas (Tabela 5) foram maiores do que da farinha crua. $\mathrm{O}$ aumento da absorção de água está relacionado com capacidade de hidratação e porosidade interna da amostra, disponibilidade de grupos hidrofílicos aptos a se ligar às moléculas de água $\mathrm{e}$ capacidade de formação de gel das moléculas de amido. As modificações ocasionadas em função da temperatura, pressão e umidade utilizadas no processo de extrusão proporcionaram um aumento da afinidade da farinha extrusada pela água. $\mathrm{O}$ aumento da solubilidade verificado está associado às alterações nas propriedades físico-químicas das proteínas, sua capacidade de interação com água e demais constituintes da farinha. Além disso, está associada às alterações na dispersão das moléculas de amilose e amilopectina, como consequência da gelatinização ou formação de compostos de baixo peso molecular.

Ainda na Tabela 5, estão apresentados os resultados referentes à atividade emulsificante da farinha extrusada da bandinha de feijão. Os tratamentos 1 e 2 apresentaram atividade emulsificante inferior aos apresentados pela farinha crua (46,43\%), enquanto que o tratamento 3 apresentou valor superior à crua. A redução da atividade emulsificante pode ser associada à alta umidade das amostras, o que torna o processo de extrusão mais severo. Neste caso, a temperatura, umidade e pressão durante o processo de extrusão alterou a quantidade de grupos hidrofilicos e hidrofóbicos disponíveis, alterando a capacidade desses constituintes de promoverem a redução da tensão superficial na interface óleo/água. Em relação à estabilidade, evidenciou-se uma acentuada diminuição na estabilidade da emulsão das farinhas extrusadas. Os resultados demonstram que o aumento da umidade e temperatura teve um efeito negativo na estabilidade da emulsão, como pode ser visto no tratamento 2 . As farinhas extrusadas não apresentaram atividade espumante, provavelmente em função da desnaturação e agregação proteica ocasionada durante o processo de extrusão. Em relação à formação de gel, as farinhas extrusadas foram capazes de gelificar a partir de concentrações de 8\% (p/v). Quando comparadas com a farinha crua, apresentaram uma melhora nesta propriedade uma vez que a farinha crua gelifica a partir de suspensões com concentração de $14 \%(\mathrm{p} / \mathrm{v})$.

\section{ANÁLISES FÍSICO-QUÍMICAS}

A composição centesimal, composição em ácidos graxos, teor de vitaminas e minerais da farinha extrusada da bandinha de feijão pode ser visualizada nas Tabelas 6, 7 e 8. Conforme a recomendação diária de ingestão de nutrientes, estabelecida pela ANVISA uma porção de $100 \mathrm{~g}$ de farinha de bandinha de feijão é capaz de suprir 36\% das necessidades de proteína, $50 \%$ de ferro e $10 \%$ de cálcio, ou seja, a inclusão dela em preparações alimentícias é viável do ponto de vista nutricional.

A amostra analisada apresentou atividade de água de $0,534( \pm 0,003)$. Sabendo-se que os valores mínimos de aw, necessários para desenvolvimento de microorganismos, variam entre 0,65 e 0,8543 , foi possível concluir que a farinha em questão apresenta atividade de água satisfatória do ponto de vista de contaminação microbiológica pois a baixa disponibilidade de água livre impedirá o crescimento de micro-organismos patógenos.

A viscosidade observada foi de $1860 \mathrm{cP}( \pm 84,85)$. O aumento da viscosidade pela adição da farinha extrusada da bandinha de feijão indica que esta pode ser eficientemente utilizada como espessante em preparações que utilizem aquecimento prévio. Este aumento da viscosidade pode ser explicado em função da pré-gelatinização dos grânulos de amido ocasionada pela extrusão. Com a abertura dos grânulos de amido, a capacidade de entumescimento e a viscosidade da solução são aumentadas quando o material é submetido a tratamento térmico.

Tabela 5. Propriedades tecnológicas da farinha de bandinha de feijão carioca extrusada.

\begin{tabular}{|c|c|c|c|c|}
\hline & Temperatura $^{\mathbf{}} \mathbf{C}$ & Umidade (\%*) & Inibidor de Tripsina & Inibidor de $\boldsymbol{\alpha}$-amilase \\
\hline 1 & 110 & 19 & $88,28(54,09 \%)$ & $91,93(75,14 \%)$ \\
\hline 2 & 120 & 19 & $76,87(62,11 \%)$ & $94,28(73,27 \%)$ \\
\hline 3 & 120 & 16 & $78,61(60,74 \%)$ & $91,11(75,82 \%)$ \\
\hline & Farinha crua & 47,75 & 69,08 & \\
\hline
\end{tabular}

Os dados apresentados correspondem à média de três determinações. Os valores entre parênteses correspondem ao aumento ocasionado na digestibilidade das amostras em relação à farinha crua. 
Tabela 6. Composição centesimal da farinha extrusada de bandinha de feijão.

\begin{tabular}{|l|l|}
\hline Determinação & Resultado \\
\hline Umidade e voláteis $(\mathrm{g} / 100 \mathrm{~g})$ & $10,10(0,04) \mathrm{a}$ \\
\hline Cinzas $(\mathrm{g} / 100 \mathrm{~g})$ & $3,96(0,03) \mathrm{a}$ \\
\hline Lipídios totais $(\mathrm{g} / 100 \mathrm{~g})$ & $2,25(0,04) \mathrm{a}$ \\
\hline Proteína* $(\mathrm{g} / 100 \mathrm{~g})$ & $18,34(0,03) \mathrm{a}$ \\
\hline Carboidratos $(\mathrm{g} / 100 \mathrm{~g})$ & $44,69 \mathrm{~b}$ \\
\hline Calorias $(\mathrm{kcal} / 100 \mathrm{~g})$ & $272 \mathrm{c}$ \\
\hline Fibra alimentar total $(\mathrm{g} / 100 \mathrm{~g})$ & $20,66(0,35) \mathrm{a}$ \\
\hline Fibra alimentar solúvel $(\mathrm{g} / 100 \mathrm{~g})$ & $4,77(0,01) \mathrm{a}$ \\
\hline Fibra alimentar insolúvel $(\mathrm{g} / 100 \mathrm{~g})$ & $15,88(0,35) \mathrm{a}$ \\
\hline
\end{tabular}

$(*)$ Fator de conversão de nitrogênio para proteína $=5,75$ a - Média e estimativa do desvio padrão. $\mathrm{b}$ - Calculado por diferença: $100-(\mathrm{g} / 100 \mathrm{~g}$ umidade $+\mathrm{g} / 100 \mathrm{~g}$ cinzas $+\mathrm{g} / 100 \mathrm{~g}$ lipídios totais $+\mathrm{g} / 100 \mathrm{~g}$ proteína $+\mathrm{g} / 100 \mathrm{~g}$ de fibra alimentar total). c - $\mathrm{O}$ valor calórico da amostra foi calculado pela soma das porcentagens de proteína e carboidratos multiplicados pelo fator $4(\mathrm{kcal} / \mathrm{g})$ somado ao teor de lipídios totais multiplicado pelo fator $9(\mathrm{kcal} / \mathrm{g})$.
Tabela 8. Conteúdo de vitaminas e minerais da farinha extrusada de bandinha de feijão.

\begin{tabular}{|l|l|}
\hline Determinação & Resultado \\
\hline Ácido ascórbico (mg/100g) & $\mathrm{ND}<1,00 \mathrm{a}$ \\
\hline Vitamina B1 - Tiamina (mg/100g) & $\mathrm{ND}<0,02 \mathrm{a}$ \\
\hline Vitamina B2 - Riboflavina (mg/100g) & $\mathrm{ND}<0,02 \mathrm{a}$ \\
\hline Vitamina B6 - Piroxidina (mg/100g) & $\mathrm{ND}<0,02 \mathrm{a}$ \\
\hline Beta caroteno (ug/100g) & $\mathrm{ND}<5,00 \mathrm{a}$ \\
\hline Vitamina A (UI/100g) & $\mathrm{ND}<3 \mathrm{a}$ \\
\hline Cálcio (mg/100g) & $101(4) \mathrm{b}$ \\
\hline Ferro (mg/100g) & $7,4(0,3) \mathrm{b}$ \\
\hline Fósforo (mg/100g) & $414(3) \mathrm{b}$ \\
\hline Potássio (mg/100g) & $1676(19) \mathrm{b}$ \\
\hline Sódio (mg/100g) & $1,6(0,1) \mathrm{b}$ \\
\hline
\end{tabular}

$\mathrm{UI}=$ Unidade Internacional; a - ND = Não detectado; b - Média e estimativa de desvio padrão

Tabela 7. Composição de ácidos graxos da farinha extrusada da bandinha de feijão.

\begin{tabular}{|l|c|c|c|}
\hline Ácidos Graxos (g/100g) & & & \\
\hline Saturados & & & 1,12 \\
\hline Monoinsaturados & & & 0,42 \\
\hline Poliinsaturaados & & & 0,62 \\
\hline Ômega 3 & & & 0,17 \\
\hline Ômega 6 & & & 0,45 \\
\hline Trans-isômeros totais & & & $<0,01$ \\
\hline & & $\%$ de Área* & $(\mathrm{g} / 100 \mathrm{~g})$ \\
\hline Composição em ácidos graxos & & 40,26 & 0,87 \\
\hline C16:0 & palmítico & 0,15 \\
\hline C18:0 & estérico & 6,87 & 0,42 \\
\hline C18:1 ômega 9 & oléico & 19,39 & 0,45 \\
\hline C18:2 ômega 6 & linoléico & 20,92 & 0,02 \\
\hline C20:0 & araquídico & 0,93 & 0,17 \\
\hline C18:3 ômega 3 & alfa linolênico & 8,07 & 0,02 \\
\hline C22:0 & behênico & 0,88 & 0,06 \\
\hline C24:0 & lignocérico & 2,68 & \\
\hline
\end{tabular}

*Área x \% lipídios $/ 100$ x F de conversão $(\mathrm{F}=0,0956)$. Limite de detecção do método = 0,01 g/100g 


\section{ANÁLISE MICROBIOLÓGICA}

Os resultados (Tabela 9) evidenciaram que não houve desenvolvimento de micro-organismos o que atesta a qualidade sanitária da farinha extrusada da bandinha de feijão.

Tabela 9. Avaliação microbiológica da farinha extrusada da bandinha de feijão.

\begin{tabular}{|l|c|c|}
\hline Determinação & Método & Resultado \\
\hline $\begin{array}{l}\text { Salmonella (em } \\
25 \text { g) }\end{array}$ & $1.1 \mathrm{e} 1.2$ & Ausente \\
\hline $\begin{array}{l}\text { Coliformes } \\
\text { termotolerantes } \\
\text { (NMP/g) }\end{array}$ & 1.3 & $<3$ \\
\hline $\begin{array}{l}\text { Estafilococos } \\
\text { coagulase positiva } \\
\text { (UFC/g) }\end{array}$ & 1.4 & $<102$ \\
\hline $\begin{array}{l}\text { Bacillus cereus } \\
\text { (UFC/g) }\end{array}$ & 1.5 & $<102$ \\
\hline
\end{tabular}

$\mathbf{N M P}=$ Número mais provável. UFC $=$ Unidades Formadoras de colônias.

\section{Conclusão}

A partir dos resultados obtidos, foi possível concluir que a produção de farinha de bandinha de feijão é viável do ponto de vista econômico e técnico. Foram realizadas 74 preparações alimentícias (Figura 1) utilizando a farinha de bandinha de feijão e todas elas tiveram boa aceitação do consumidor alvo do projeto. Diante disso, a IBIA estruturou a sua comercialização (Figura 2) o que ocasionou ampliação do seu portifólio de produtos, o aumento das receitas e geração de maior valor agregado para os residues gerados no processo de beneficiamento dos grãos de feijão. Os resultados favoráveis da execução deste projeto de inovação permitiram ressaltar a importância do Edital SENAI SESI de Inovação para transferência de tecnologia e aumento da competitividade da Indústria Brasileira.

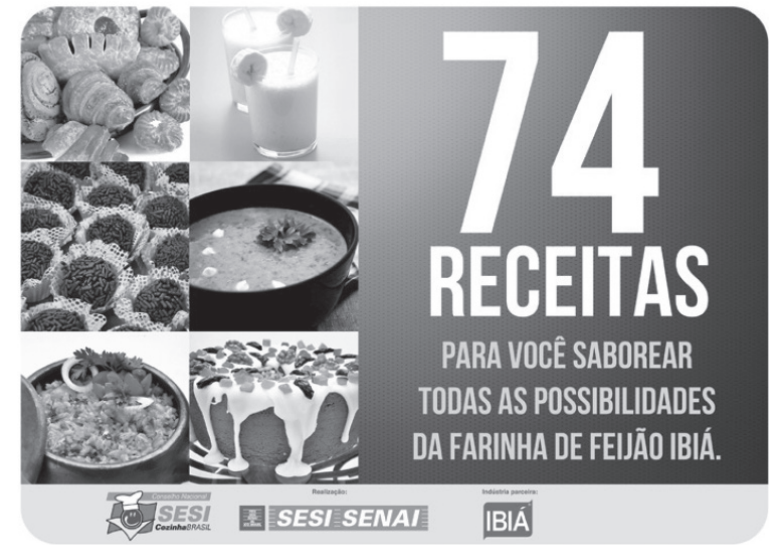

Figura 1. Preparações alimentícias elaboradadas com a farinha de bandinha de feijão. Material de divulgação do projeto Edital SENAI SESI de Inovação

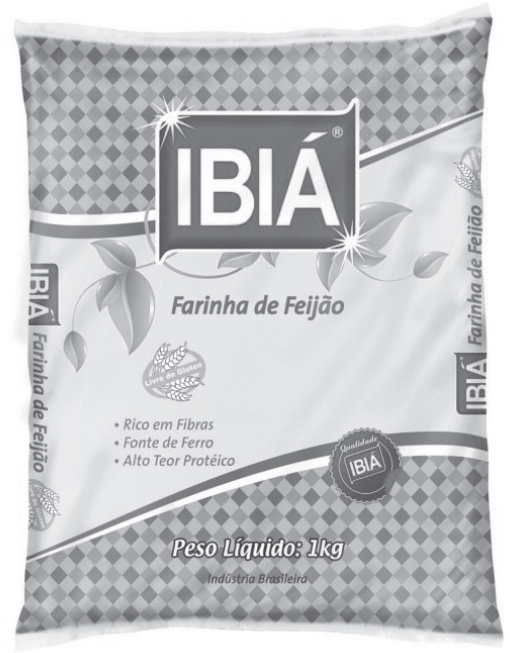

Figura 2. Apresentação comercial da farinha de bandinha de feijão.

\section{Agradecimento}

Os autores agradecem ao SENAI Departamento Nacional e ao CNPq pelo apoio financeiro. 


\section{Referências}

1. Vieira, C; Paula Júnior, T. J. P.; Borém, A. Feijão. 2 ed. Atual. Viçosa: Ed. UFV, 2006.

2. Fuente, M.D.L.; Borrajo, A.; Bermúdez, J.; Lores, M.; Alonso, J.; López, M.; Santalla, M.; Ron, A.M.D.; Zapata, C.; Alvarez, G. 2-DE-based proteomic analysis of common bean (Phaseolus vulgaris L.) seeds. Journal of Proteomics, 74, p. 262-267, 2011.

3. Canniatti Brazaca, S. G.; Rocha, L. C.; Silva, A. G.; Caracterização físico-química, digestibilidade protéica e atividade antioxidante de feijão comum (Phaseolus vulgaris L.). Alimentos e Nutrição, Araraquara, v. 20, n.4, p.591-598, out/dez. 2009.

4. Cardoso Filho, N.; Ciacco, C. F.; Sgarbieri, V. C. Efeito de algumas variáveis do processo de extrusão nas características tecnológicas da farinha do feijão comum. Ciência e Tecnologia de Alimentos, Campinas, v. 16, n. 2, p. 158-164, 1996.

5. Da Silva, E. M.; Ascheri, J. L. R.; Ascheri, D. P.; Teba, C. Da S. Efeito da extrusão termoplástica nas características de viscosidade de pasta, solubilidade e absorção de água de farinhas prégelatinizadas de milho e feijão carioca (Brs pontal). B. Ceppa, Curitiba, v. 31, n. 1, jan./jun. 2013.

6. El-Dash, A.A. Aplication and control of thermoplastic extrusion of cereals for food andindustrial uses. In: Cereals a renewable resurce: theory and practice. AmericanAssociation of Cereal Chemists, St. Paul, p.165-216, 1981.

7. Gualberto, D. G. Avaliação nutricional e sensorial de misturas de feijão e soja processados por extrusão. 1981. Dissertação (Mestrado em Ciência e Tecnologia de Alimentos) - Universidade Federal de Viçosa, Viçosa, MG.

8. Batista, K.A.; Prudêncio, S.H.; Fernandes, K.F. Changes in the functional properties and antinutritional factors of extruded hardto-cook common beans Journal of Food Science, 75, p. 286-290, 2010.

9. Kunitz, M. Crystalline soybean trypsin inhibitor. II General Properties. The Journal of General Physiology, 30, p. 291-310, 1947.

10. Arnon, R., Papain, in Methods in Enzymology, G. Perlmann; D.L. Loran, Editors. 1970, Academic Press: New York. p. 226-244.

11. Deshpande, S.S.; Sathe, S.K.; Salunkhe, D.K.; Comforth, D.P. Effects of dehulling on phytic acid, polyphenols, and enzyme inhibitors of dry beans (Phaseolus vulgaris L.). Journal of Food Science, 47, p. 1846-1850, 1982.

12. Miller, G.L. Use of dinitrosalicylic acid reagente for determination of reducing sugar. Analytical Chemistry, 31, p. 426-428, 1959.

13. Moreira, R.A.; Perrone, J.C. Purification and partial characterization of a lectin from Phaseolus vulgaris. Plant Physiology, v. 59, p. 783-787, 1977.

14. Hsu, H.W.; Vavak, D.L.; Satterlee, L.D.; Miller, G.A. A multienzyme technique for estimating protein digestibility.
Journal of Food Science, 42, p. 1269-1273, 1977

15. Okezie, B.O.; Bello, A.B. Physicochemical and functional properties of winged bean flour and isolate compared with soy isolate. Journal of Food Science, 53, p. 450-454, 1988.

16. Yasumatsu, K.; Sawada, S.; Moritaka, S.; Misaki, M.; Toda, J.; Wada, T.; Ishii, K. Whipping and emulsifying properties of soybean products. Agricultural and Biochemical Chemistry, 36, p. 719-727, 1972

17. Horwitz, W. (Ed.) Official methods of analysis of AOAC International. 18th ed., Gaithersburg, Maryland, 2005. Current through Revision 1, 2006. Cap. 45, met.985.29, p.97-98.

18. Zenebon, Odair; Pascuet, Neus Sadocco (Coord.). Métodos físicoquímicos para análise de alimentos. 4 ed. Brasília: Ministério da Saúde/ANVISA; São Paulo: Instituto Adolfo Lutz, 2005. cap. 4, met. 034B, p. 119.

19. Prosky, L.; Asp, N-G; Furda, I.; Devries, J.W.; Schweizer, T.F. \& Harland, B.F. Determination of total dietary fiber in foods, food products and total diets: Interlaboratorial Study. Journal of The Association Official Analytical Chemists, Arlingtton, v.67, n.6, p.1044-1052, 1984.

20. Kalil, A.. Manual Básico de Nutrição. São Paulo: Instituto de Saúde, 1975.

21. Passmore, R.; Nicol, B.M.; Rao, M.N. Manual Sobre Necessidades Nutricionales Del Hombre. Ginebre: O.M.S., 1975. (Série de Monografia, 61).

22. United States Department Of Agriculture. Composition of Foods. Agriculture Handbook $N^{\circ} 8$, WASHINGTON; USDA, 1963.

23. Firestone, D. (Ed.). Official methods and recommended practices of the American Oil Chemists Society. 6th ed. 2009. Urbana: AOCS, 2009. Met. Ce 1f - 96, Ce 1-62.

24. Horwitz, W. Official Methods of Analysis of the Association of Official Analytical Chemists. 18th Ed. 2005. Current Through Revision 3, 2010 Gaithersburg, Maryland, AOAC, 2010. Chapter 41 Met. 996.06, p. 20.

25. Hartman, L.; Lago, R.C.A. Rapid preparation of fatty acid methyl esters from lipids. Lab. Practice, v. 22, n. 8, p. 475-476, 1973.

26. Food Standards Agency. Mc Cance and Widdowson's The Composition of Foods, Sixth Summary Edition. Cambridge:2002, Royal Society of Chemistry. $537 \mathrm{p}$.

27. Carvalho, P.R.N.; Collins, C.A.; Rodriguez-Amaya, D.B. Comparison of provitamin A determination by normal-phase gravity-flow column chromatography and reversed phase high performance liquid chromatography. Chromatographia, Wiesbaden, v. 33, p. 133-37, 1992.

28. Horwitz, W. (Ed.). Official methods of analysis of the Association of Official Analytical Chemists. 18th ed. Gaithersburg, Maryland: AOAC, 2005. Chapter 45, methods 961.15, p.67-69. Current Through Revision 3, 2010.

29. Gregory, J.F.; Kirk, J.R. Assessment of stocage effects on vitamin B6 stability and bioavailability in dehydrated food systems. 
Journal of Food Science, Cicago, v. 43, p. 1801-09, 1978.

30. Horwitz, W. (ED.). Official methods of analysis of the Association of Official Analytical Chemists. 18th ed. Gaithersburg, Maryland: AOAC, 2005. Chaper 45, methods 942.23, p. 11-13. Current Through Revision 3, 2010.

31. Van De Weerdhof, T.; Wiersun, M.L.; Reissenwebweber, H.. Application of liquid chromatography in food analysis. Journal Chromatography, Amsterdam, v. 83, p. 455-60, 1973.

32. Horwitz, W. (Ed.). Official methods of analysis of the Association of Official Analytical Chemists. 18th ed. Gaithersburg, Maryland: AOAC, 2005. Chapter 45, methods 970.65, p. 14-16. Current Through Revision 3, 2010.

33. Van De Weerdhof, T.; Wiersun, M.L.; Reissenwebweber, H.. Application of liquid chromatography in food analysis. Journal Chromatography, Amsterdam, v. 83, p. 455-60, 1973.

34. Arakawa, N.; Otsuka, M.; Kurata, T.; Inagki, C. Separative determination of ascorbic acid and erythorbic acid by highperformance liquid chromatography. Journal Nutritional Science Vitaminology, Japan, v. 27, p. 1-7, 1981.

35. Horwitz, W. (Ed) Official methods of analysis of the Association of Official Analytical Chemists. 18th ed. Gaithersburg, Maryland: AOAC, 2005. Current Through Revision 3, 2010. cap. 50, met. 985.35 e 984.27 , p.15-18.

36. Slavin, S.; Petersen, G.E.; Lindhal, P.C. Determination of heavy metals in meats by atomic absorption spectroscopy. Atomic Absorption Newsletter, Norwalk, v. 14, n. 3, p. 57-59, 1975.

37. Brasil. Agência Nacional de Vigilância Sanitária. Resolução da Diretoria Colegiada RDC-12, 2001. Disponível em http://www. anvisa.gov.br/legis/resol/12_01rdc.htm.

38. ISO 6579. Microbiology of food and animal feeding stuffs Horizontal method for the detection of Salmonella spp., 4th ed. The International Organization for Standardization, 2002, Corrigendum 1:2004, Amendment 1:2007.

39. AOAC Official Method 2003.09 (Salmonella PCR Bax System). In: HORWITZ, W. (ed.), Official Methods of Analysis of AOAC International, 18th ed. Gaithersburg, Maryland: AOAC International, 2005. Chapter 17, p.194.

40. ISO 7251. Microbiology of food and animal stuffs - Horizontal method for the detection and enumeration of presumptive Escherichia coli - most probable number technique, $3 \mathrm{rd}$ ed. The International Organization for Standardization, 2005.

41. ISO 6888-1. Microbiology of food and animal feeding stuffs Horizontal method for the enumeration of coagulase-positive staphylococci (Staphylococcus aureus and other species) - Part 1: Technique using Baird-Parker agar medium, 1st ed. The International Organization for Standardization, 1999, Amendment $1: 2003$.

42. Downes, F. P. \& Ito, K. (eds.). Compendium of Methods for the Microbiological Examination of Foods, 4th ed. Washington: American Public Health Association, 2001. 676 p.
43. Beauchat, L. R. Influence of aw on growth, methabolic, activities and survival of yeasts and molds. Journal of Food Protect, Ames, 46: 135-41, 1983.

\section{Christiane A. Starling ; Fernanda G.de Souza \& Adriana A. de Carvalho}

Instituto SENAI de Tecnologia em Alimentos e Bebidas. Escola SENAI Vila Canaã. Rua Professor Lázaro Costa 348, Vila Canaã, CEP 74.415420, Goiânia, Goiás, Brasil.

*E-mail: Christiane.senai@sistemafieg.org.br 